\title{
Sexo del hospedero y dosis infectante de parásitos como factores en el desarrollo de la infección con Trypanosoma cruzi en un modelo murino
}

\author{
CONSTANZA URZÚA* , MARÍA ANGÉLICA MORALES*, ULISES VERGARA* \\ MARÍA TERESA PALAU* y CLAUDIO ZÚÑIGA*
}

\section{SEX OF HOST AND INITIAL DOSE OF INOCULUM LIKE FACTORS IN THE DEVELOPMENT OF THE INFECTION WITH Trypanosoma cruzi IN A MURINE MODEL}

Male and female mice of ACA strain were infected with 200 and 2,000 bloodstream trypomastigotes from Dm 28 c clone of Trypanosoma cruzi. All the animals were susceptible to the infection regardless of their sex and dose of initial inoculum used. Both male and female, infected with 2,000 parasites showed significantly higher level of parasitemia than mice infected with the lower dose. At the same time, males developed higher parasitemia level than females, when they were infected with both 200 and 2,000 parasites. Despite of experimental animals behaved as susceptible, the survival period was significantly longer in males infected with 200 trypomastigotes when compared to males infected with 2,000 parasites. Females infected with 2,000 survive a longer time than males infected with the same dose of parasites. No significant differences were observed during the survival period when comparing females infected with 200 and 2,000 parasites, neither in the case of male and female infected with 200 parasites. Apparently, the parasitemia level is the main characteristic affected by the variables sex of the host and initial parasite dose.

Key words: Experimental Chagas'disease, Trypanosoma cruzi, Murine trypanosomiosis.

\section{INTRODUCCIÓN}

El parásito Trypanosoma cruzi causa la enfermedad de Chagas, en el humano, un sindrome debilitante crónico e incurable que afecta a millones de personas en Latinoamérica ${ }^{1}$. La principal vía de transmisión del parásito a los mamíferos es mediante insectos hematófagos de la familia Reduviidae, que al igual que T.cruzi sólo existen en Latinoamérica ${ }^{2}$. Se postula que la infección de animales domésticos, como perros y gatos, ocurriría principalmente por vía oral, con deposiciones del vector contaminadas presentes en el pelaje o alimentos ${ }^{3,4}$. De la misma manera, los pequeños roedores, importantes reservorios naturales, se infectarían principalmente por consumir insectos infectados ${ }^{5}$. La infección experimental por T.cruzi se ha reproducido, fundamentalmente la etapa aguda, en diversas especies animales, como ser conejos ${ }^{6}$, perros $^{7}$,

\footnotetext{
* Departamento de Medicina Preventiva Animal, Facultad de Ciencias Veterinarias y Pecuarias, Universidad de Chile. Casilla 2 ,Correo 15, La Granja, Santiago, Chile. E-mail: clzuniga@uchile.cl

Financiado por SIDA / SAREC.
} 
ratones $^{8}$ y primates no humanos ${ }^{9}$. En el modelo murino, distintas cepas puras de ratones difieren en la susceptibilidad o resistencia a la infección y se plantea un complejo control génico de los niveles de parasitemia y la supervivencia de los animales infectados ${ }^{10}$. Múltiples factores tanto dependientes del hospedero como del parásito, se encuentran involucrados en la determinación de la resistencia o susceptibilidad a la infección con T. cruzi. En el hospedero, factores genéticos asociados al Complejo Mayor de Histocompatibilidad parecen importantes para determinar el desarrollo de la infección ${ }^{11}$.

En el presente trabajo se analizó el efecto del sexo de los individuos infectados y la dosis infectante de parásitos en ratones machos y hembras de la cepa ACA, infectados con $200 \mathrm{y}$ 2.000 trypomastigotes sanguíneos del clon Dm 28c de T. cruzi.

\section{MATERIAL Y MÉTODO}

Ratones: Se utilizaron ratones de la cepa ACA $\left(\mathrm{H} 2^{\mathrm{f}}\right)^{12}$. Esta cepa proviene originalmente del Jackson Laboratory, Maine, U.S.A. y es mantenida por cruzamientos endogámicos en el bioterio de la unidad de Inmunología, Fac. Cs. Veterinarias, Universidad de Chile.

Parásitos: Las infecciones experimentales se llevaron a cabo utilizando tripomastigotes sanguíneos del clon Dm 28c de T. cruzi ${ }^{13}$. Esta cepa de parásito es mantenida en el bioterio de la unidad de Inmunología por pasajes sucesivos en ratones $\mathrm{Balb} / \mathrm{c}$.

Modelos de infección: Dos grupos de 10 hembras y 10 machos, ambos de 10 semanas de edad fueron inoculados intraperitonealmente (i.p.) con 200 parásitos / ratón. Otros dos grupos, 10 hembras y 10 machos, fueron inoculados i.p. con 2000 parásitos / ratón. Como los inóculos son diluciones de sangre con parásitos, como grupo control se inocularon 10 hembras y 10 machos con la misma dilución de sangre $\mathrm{Balb} / \mathrm{c}$ de ratones sin infectar, para descartar posibles fenómenos adversos inducidos por la sangre Balb/c.

Estudio de parasitemia: Cada dos días, luego de la inoculación inicial, los niveles de parasitemia se analizaron hasta que los animales se hicieron negativos a la presencia de parásitos sanguíneos. El recuento de parásitos se realizó en muestras de sangre de cada animal tomadas en tubos de microhematocrito heparinizados. La cantidad de parásitos en cada muestra se determinó según el método descrito por Arias y Ferro (1988) ${ }^{14}$. Los resultados del número de parásitos obtenidos en cada día de estudio se expresaron como el promedio de parasitemia del grupo más la desviación estándar correspondiente.

Análisis estadístico: Los resultados de los niveles máximos de parasitemia fueron analizados mediante un análisis de varianza para un experimento de dos factores en un diseño factorial, considerando los factores sexo y dosis de inóculo inicial. El análisis de supervivencia se hizo de acuerdo al método de Kaplan y Meier ${ }^{15}$.

\section{RESULTADOS}

En la Figura 1 se muestra la evolución de la parasitemia en ratones machos infectados con 200 y 2.000 parásitos. El período de prepatencia fue de 4 días post-infección (p.i), en ambos casos. Al usar un inóculo de 200 parásitos se alcanzó un nivel máximo de parasitemia de $6,1 \times 10^{5}$ parásitos/ml que resultó ser significativamente menor $(\mathrm{p}<0,05)$ que el nivel máximo de $2,2 \mathrm{x}$ $10^{6}$ parásitos $/ \mathrm{ml}$ obtenido al infectar con 2.000 parásitos. La Figura 2 muestra el desarrollo de la parasitemia en ratones hembras infectadas con 200 y 2.000 parásitos. Para el grupo infectado con 200 parásitos el nivel máximo de parasitemia se alcanzó al día 8 p.i. y fue de 4,5 x $10^{5}$ parásitos $/ \mathrm{ml}$ y el grupo infectado con 2.000 , presentó su nivel máximo al día 10 p.i. con 1,4 x $10^{6}$ parásitos/ ml. Estas diferencias en los niveles máximos de parasitemia fueron también estadísticamente significativas $(p<0,05)$. En la Figura 3 se

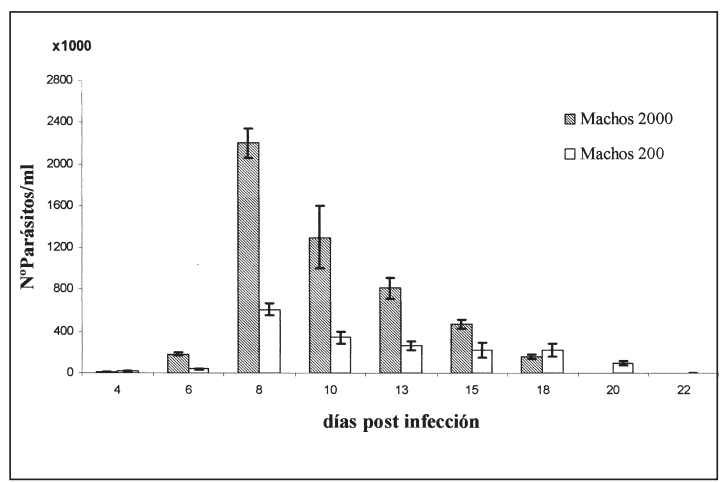

Figura 1. Niveles de parasitemia de ratones machos de la cepa ACA infectados con 200 y 2.000 trypomastigotes sanguíneos del clon Dm 28c de T. cruzi. 
compara el desarrollo de la parasitemia en machos y hembras infectados con 200 parásitos. No se observan diferencias en la prepatencia, duración de la parasitemia y día en que se alcanzó el nivel máximo pero sí existe una diferencia significativa en los niveles máximos alcanzados, puesto que los machos presentaron un valor de $6,1 \times 10^{5} \mathrm{y}$ las hembras $4,5 \times 10^{5}$ parásitos $/ \mathrm{ml}(\mathrm{p}<0,05)$. Una situación similar se observa al comparar los niveles de parasitemia en machos y hembras infectados con 2.000 parásitos (Figura 4). Los machos muestran un nivel máximo de parasitemia significativamente más alto que las hembras $(\mathrm{p}<$ $0,05)$.

La comparación en la supervivencia de los diferentes grupos de animales infectados se muestra en las Figuras 5 a 8. La Figura 5 muestra

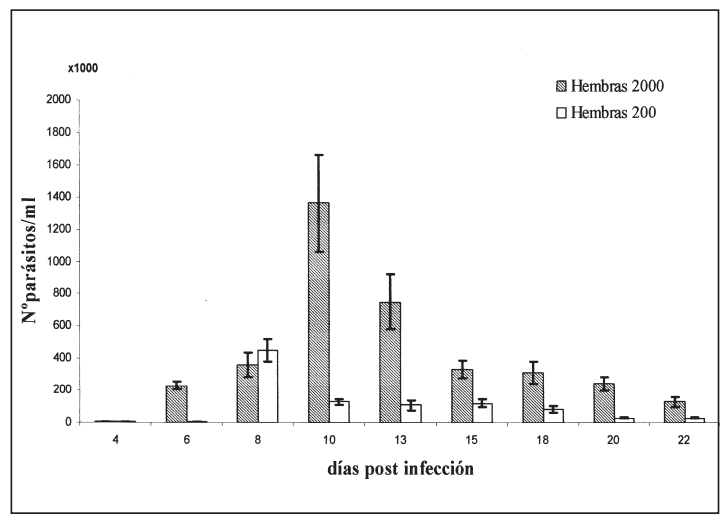

Figura 2. Niveles de parasitemia de ratones hembras de la cepa ACA infectados con 200 trypomastigotes sanguíneos del clon Dm 28c de T. cruzi.

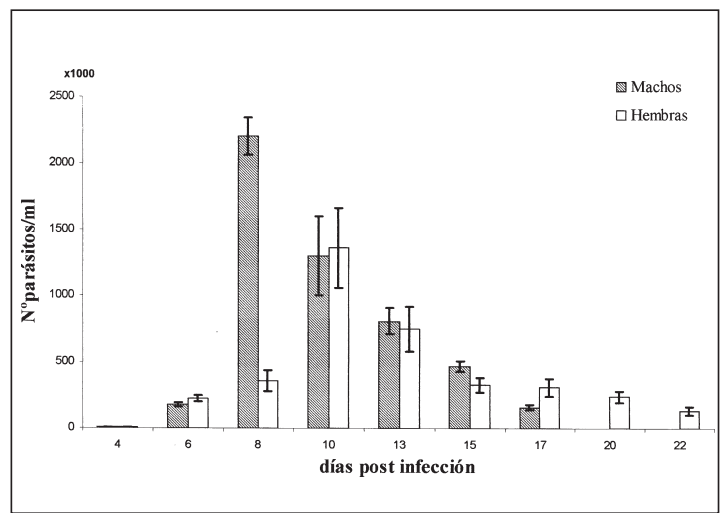

Figura 4. Niveles de parasitemia de ratones machos y hembras de la cepa ACA infectados con 2000 trypomastigotes sanguíneos del clon Dm 28c de T. cruzi. la sobrevivencia de ratones machos infectados con 200 y 2.000 parásitos. En los ratones infectados con 200 parásitos el $100 \%$ de mortalidad acumulada se alcanzó al día 28 p.i. mientras que al infectar con 2.000, esto ocurrió al día 20 p.i. El análisis estadístico de los tiempos de sobrevida indicó que el valor de chi cuadrado $\left(X^{2}\right)$ para estos dos grupos correspondió a 6,3, teniendo una diferencia significativa a nivel de $5 \%$. En la Figura 6 se puede observar la supervivencia de las hembras infectadas con ambas dosis de parásitos. En las hembras infectados con 2.000 parásitos el $100 \%$ de mortalidad se alcanzó a los 24 días p.i. mientras que en las hembras infectadas con 200 parásitos se obtuvo un $70 \%$ de mortalidad al día 32 p.i. (el $30 \%$ de los animales sobrevivió hasta 6 meses

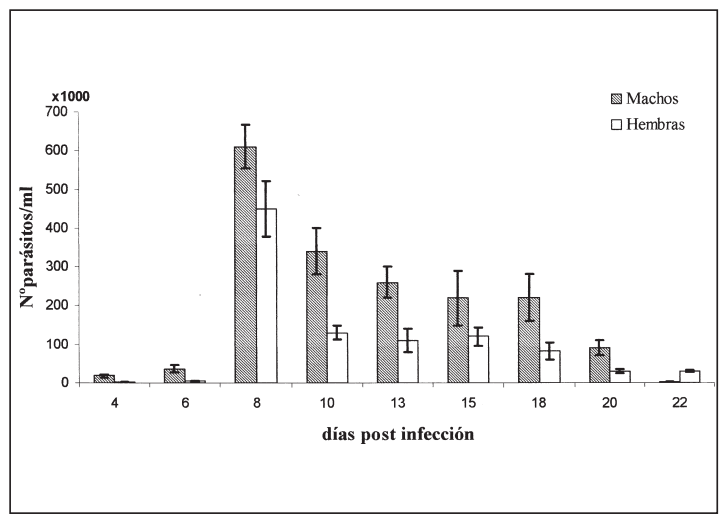

Figura 3. Niveles de parasitemia de ratones machos y hembras infectados con 200 trypomastigotes sanguíneos del clon Dm 28c de T. cruzi.

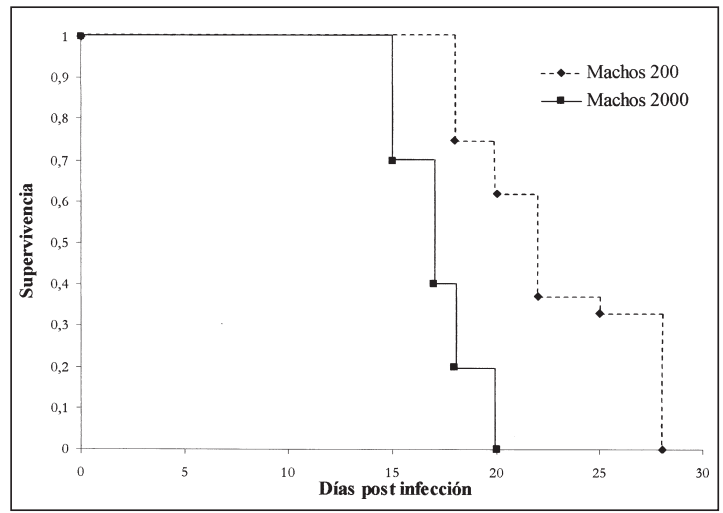

Figura 5. Supervivencia de ratones machos de la cepa ACA infectados con 200 y 2.000 trypomastigotes sanguíneos del clon Dm 8c de T. cruzi. 


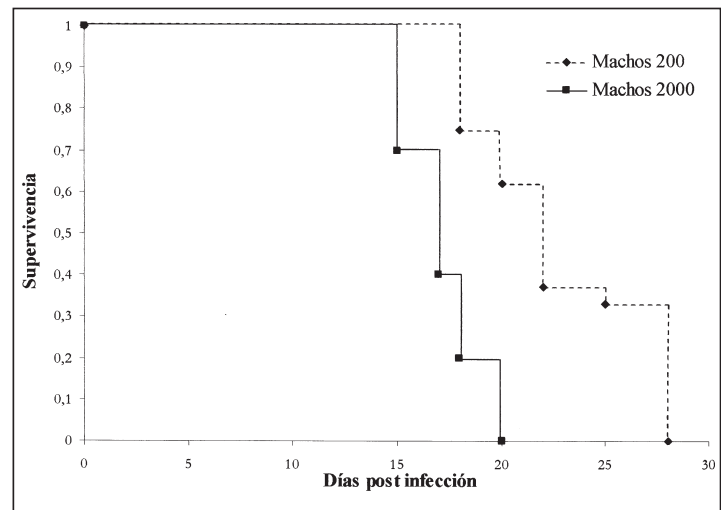

Figura 6. Supervivencia de ratones hembras de la cepa ACA infectados con 200 y 2.000 trypomastigotes sanguíneos del clon Dm 28c de T. cruzi.

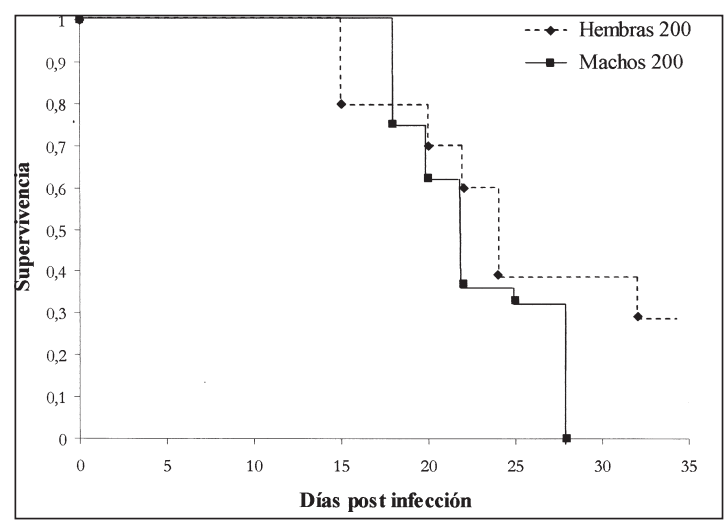

Figura 8. Supervivencia de ratones machos y hembras de la cepa ACA infectados con 2.000 trypomastigotes sanguíneos del clon Dm 28c de T. cruzi.

p.i., momento en que fueron sacrificados).

A pesar de lo anterior, la comparación de la mortalidad acumulada en los dos grupos mostró un valor estadístico de $X^{2}$ de 2,4, indicando que las diferencias no son significativas. En la Figura 7 se muestra la comparación de la supervivencia de hembras y machos infectados con 200 parásitos. El valor de $X^{2}$ obtenido en relación a estos dos grupos fue de 1,2 lo que indica que las diferencias no son significativas. Por último, en la Figura 8 se observa la comparación de hembras y machos infectados con 2000 parásitos. En este caso el valor de $X^{2}$ fue de 6,4 indicando una diferencia significativa a nivel de $5 \%$ entre ambos grupos.

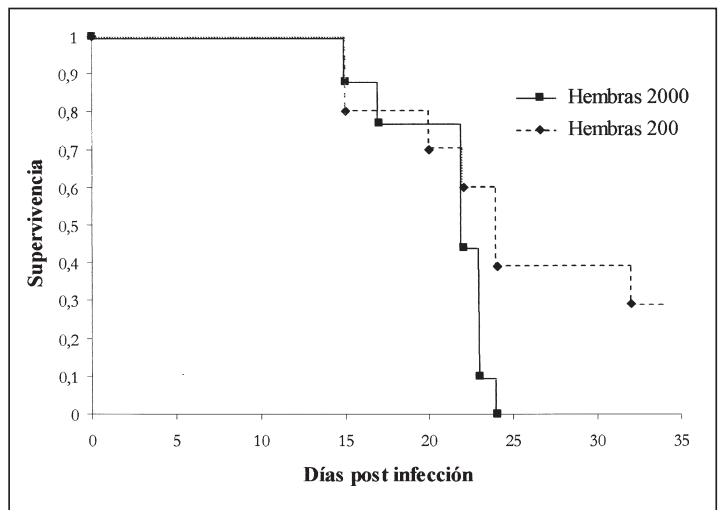

Figura 7. Supervivencia de ratones machos y hembras de la cepa ACA infectados con 200 trypomastigotes sanguíneos del clon Dm 28c de T. cruzi.

\section{DISCUSIÓN}

La cepa de ratones ACA, que posee el haplotipo $\mathrm{H} 2^{\mathrm{f}}$ es considerada como susceptible a la infección con distintos aislados de $T$. cruzi $i^{11}$. Los resultados del presente estudio confirman esta sugerencia puesto que independientemente del sexo o la dosis de inóculo, los animales se comportaron como altamente susceptibles a la infección con el clon Dm 28c de T.cruzi, aún cuando la dosis infectante de parásitos fue inferior a la utilizada en estudios anteriores ${ }^{11}$. Nuestros resultados difieren de trabajos anteriores que clasifican al clon Dm 28c como un aislado medianamente virulento ${ }^{13,16}$. Sin embargo, estos trabajos previos se realizaron en ratones Balb/c infectados con tripomastigotes originados en cultivo axénico y no con tripomastigotes sanguíneos, como en el presente trabajo. Nuestros resultados apoyan la idea que la susceptibilidad o resistencia, al menos en el modelo murino, no son fenómenos generales sino que están habitualmente referidos a una cepa de ratón y/o una cepa de parásito en particular.

Los resultados obtenidos indican que el número de parásitos que ingresan originalmente al organismo modifican el curso de la infección con T.cruzi afectando fundamentalmente los niveles de parasitemia y el período de supervivencia de los animales infectados. Tanto hembras como machos infectados con 2.000 parásitos alcanzaron niveles de parasitemia significativamente más altos que los animales infectados con la dosis menor y que con esta misma dosis los machos presentan niveles más 
altos que las hembras. En humano, este aspecto es muy poco conocido, debido a que habitualmente los pacientes estudiados ya están en la etapa crónica, con ausencia de parasitemia evidente.

Estudios previos en diversos modelos experimentales sugieren que los machos son más susceptibles que las hembras ${ }^{17-19}$. Sin embargo, los resultados del presente estudio parecen apoyar la idea que aún cuando los niveles de parasitemia son más altos en machos que en hembras infectadas, no se llega al extremo de resistencia en las hembras y susceptibilidad en los machos, como ocurre en ratas de la cepa F344 en las cuales la infección con T.cruzi produce la muerte sólo de los machos ${ }^{20}$.

En el presente trabajo, al comparar el tiempo de supervivencia de los animales infectados, se observaron diferencias significativas entre los machos inoculados con 200 y 2.000 parásitos y por lo tanto los animales infectados con una dosis menor sobreviven un tiempo más prolongado. Por otro lado, al comparar hembras y machos infectados con 2.000 parásitos se observó que las hembras sobreviven más tiempo. El período de supervivencia no fue significativamente distinto entre las hembras infectadas con $200 \mathrm{y}$ 2.000 parásitos ni tampoco en el caso de hembras y machos inoculados con 200 tripomastigotes. A pesar que diversos trabajos han tratado de asociar la susceptibilidad a la infección con $T$. cruzi con los niveles de hormonas sexuales, no se han reportado hasta ahora resultados definitivos. Estudios previos, utilizando ratones de la cepa de $\mathrm{CFI}^{21}$ y hembras del roedor Calomys callosus ${ }^{18}$ han demostrado que las hembras ovariectomizadas presentan mayores niveles de parasitemia y mayor mortalidad acumulada al ser infectadas con la cepa Brazil y cepa Y de $T$. cruzi, respectivamente, en relación a hembras controles. Se ha sugerido que las hormonas sexuales tendrían un efecto importante en la infección con T.cruzi y que la función inmunoinhibidora de la testosterona sería responsable de la diferente susceptibilidad de machos y hembras. Sin embargo, se ha descrito que al infectar grupos de ratones Balb/c, los machos dominantes presentan mayores niveles de testosterona y menores niveles de parasitemia que los machos en inferior posición social, los cuáles presentan menores niveles de testosterona y mayores niveles de parasitemia ${ }^{22}$. Todo parece indicar que la resistencia o susceptibilidad a la infección con T. cruzi no es consecuencia de algo tan simple como diferencias en los niveles de una hormona u otra, sino que debe existir una compleja regulación neuro-endocrina-inmunológica en el control de los niveles de parasitemia y la susceptibilidad o resistencia a la infección.

\section{RESUMEN}

Se infectaron ratones de la cepa ACA, machos y hembras, con 200 y 2.000 tripomastigotes sanguíneos del clon Dm 28c de Trypanosoma cruzi. Todos los animales se comportaron como susceptibles a la infección, en forma independiente del sexo y la dosis de inóculo inicial. Los animales, tanto machos como hembras, infectados con 2.000 parásitos presentaron niveles de parasitemia significativamente más altos que los ratones infectados con la dosis menor. A su vez, los machos desarrollaron niveles de parasitemia más altos que las hembras, tanto al infectar con 200 como con 2.000 parásitos. A pesar de comportarse todos los animales experimentales como susceptibles, el período de supervivencia fue significativamente más prolongado en los machos infectados con 200 comparado con lo ocurrido con los machos infectados con 2.000 parásitos. Las hembras infectadas con 2.000 parásitos sobreviven más tiempo que los machos infectados con la misma dosis. No se observaron diferencias significativas en el tiempo de supervivencia, al comparar hembras infectadas con ambas dosis ni tampoco al comparar machos y hembras infectados con 200 parásitos. Al parecer, en nuestro caso, la principal característica afectada por las variables sexo y dosis de inóculo inicial es el nivel de parasitemia.

\section{REFERENCIAS}

1.- DÍAS J C P, SILVEIRA A, SCHOFIELD C J. The impact of Chagas disease Chagas disease control in Latin America -A review. Mem Inst Oswaldo Cruz 2002; 97 : 603-12.

2.- STEVENS J, NOYES H, SCHOFIELD $C$ et al.The molecular evolution of Trypanosomatidae. Adv Parasitol 2001; 48: 1-53.

3.- SCHOFIELD C J. Trypanosoma cruzi the vectorparasite paradox. Mem Inst Oswaldo Cruz 2000; 4: 535-44.

4.- MONTENEGRO V, JÍMENEZ M, PINTO-DÍAS J et al. Chagas disease in dogs from endemic areas of Costa Rica. Mem Inst Oswaldo Cruz 2002; 97: 491-4.

5.- DIOTAIUT L, PEREIRA A, LOIOLA $\mathrm{C}$ et al. Interrelation of sylvatic and domestic transmission of 
Trypanosoma cruzi in areas with and without domestic vectorial transmission in Minas Gerais,Brazil. Mem Inst

Oswaldo Cruz 1995; 90: 443-8.

6.- TEXEIRA A R, FIGUEIREDO F, REZENDE $\mathrm{F}$ et al. Chagas' disease: A clinical parasitology, immunological and pathological study in rabbits. Am J Trop Med Hyg 1983; 32: 258-72.

7.- LANA M, CHIARI E, TAFURI W. Experimental Chagas' disease in dogs Mem Inst Oswaldo Cruz 1992; 87: 59-71.

8.- ZÚÑIIGA C, VARGAS R, VERGARA U. Evolución de la infección con Trypanosoma cruzi en cepas susceptibles y resistentes de ratones. Arch Med Vet 2002; 2: 183-8.

9.- ROSNER J, SCHININI A, ROVIRA T et al. Acute Chagas'disease in non human primates. Trop Med Parasitol 1998; 39: 51-5.

10.- TRISCHMANN T, BLOOM B. Genetics of murine resistance to Trypanosoma cruzi. Infect Immun 1982; 35: 546-51.

11.- JURI M, FERREIRA A, RAMOS A et al. Non-lytic antibodies in $\mathrm{H}-2$ controlled resistance to acute infection with Trypanosoma cruzi. Braz J Med Biol Res 1990; 23: 685-95.

12.- KLEIN J, FIGUEROA F, DAVID C. H-2 Haplotypes. Genes and antigens: second listing. Inmunogenetics 1983; 17: 533-96.

13.- CONTRERAS V, ARAUJO - JORGE T, BONALDO M et al. Biological aspect of the Dm 28c clone of Trypanosoma cruzi after metacyclogenesis in chemically defined media. Mem Inst Oswaldo Cruz 1988; 83: 123-33.
14.- ARIAS A, FERRO E. Quantification of Trypanosoma cruzi parasitemia by direct micromethod. Trans Roy Soc Trop Med Hyg 1988; 82: 248.

15.- KAPLAN E, MEIER P. Non parametric estimation from incomplete observation. JASA 1958; 53: 457-81.

16.- SÁNCHEZ G. Estudio de la variabilidad en poblaciones y clones de Trypanosoma cruzi por criterios biológicos, inmunológicos y bioquí-micos.1992. Tesis para obtener Doctorado en Ciencias. Facultad de Ciencias, Universidad de Chile, Santiago, Chile.

17.- HAUSCHKA T. Sex of the host as factor in Chagas disease. J Parasitol 1947; 33: 399-405.

18.- PRADO J C, LEAL M, ANSELMO-FRANCI J et al. Influence of female gonadal hormones on the parasitemia of female Calomys callosus infected with the Y strain of Trypanosoma cruzi. Parasitol Res 1997; 2: $100-5$.

19.- DE SOUZA E M, RIVERA M, ARAUJO - JORGE T et al. Modulation induced by estradiol in the acute phase of Trypanosoma infection in mice. Parasitol Res 2001; 7: 513-20.

20.- RIVERA-VANDENPAS M, RODRÍGUEZ A, AFCHAIN D. Trypanosoma cruzi variation in susceptibility of inbred strains of rats. Acta Trop 1983; 40: 5-10.

21.- CHAPMAN W, HANSON W, WAITS V. The influence of gonadectomy of host on parasitemia and mortality of mice infected with Trypanosoma cruzi. J Parasitol; 2: 213-6.

22.- SCHUSTER J P,SCHAUB G. Experimental Chagas disease: the influence of sex and psychoneuroimmunological factors. Parasitol Res 2001; 12: 9941000 .

\section{Informaciones sobre el XVII Congreso Latinoamericano de Parasitología}

9 al 12 de noviembre, 2005 - Hotel Costa Galana

Mar del Plata, Buenos Aires, Argentina

\section{Informaciones:}

Lab. de Zoonosis Parasitarias, Dto. Biología. Fac. Cs. Ex. y Nat., UNMdP

Funes 3250 (7600), Mar del Plata, Argentina

Te: 5402234752426 int 450, Fax: 5402234753150

e-mail: flapxvii@mdp.edu.ar 